\title{
Probabilistic Planning with Preferences over Temporal Goals
}

\author{
Jie $\mathrm{Fu}^{1}$
}

\begin{abstract}
We present a formal language for specifying qualitative preferences over temporal goals and a preferencebased planning method in stochastic systems. Using automatatheoretic modeling, the proposed specification allows us to express preferences over different sets of outcomes, where each outcome describes a set of temporal sequences of subgoals. We define the value of preference satisfaction given a stochastic process over possible outcomes and develop an algorithm for time-constrained probabilistic planning in labeled Markov decision processes where an agent aims to maximally satisfy its preference formula within a pre-defined finite time duration. We present experimental results using a stochastic gridworld example and discuss possible extensions of the proposed preference model.
\end{abstract}

\section{INTRODUCTION}

In an uncertain environment, preference plays a key role in human's decision-making that decides what constraints to satisfy when not all constraints can be achieved [1]. To achieve such cognitive flexibility in autonomous systems, it is necessary to investigate the model of preferences and the planning problems given preferences in deterministic, probabilistic, or even adversarial environments. In this paper, we focus on the specification of preferences over temporal goals, and the design of preference-based policies in a discrete stochastic system modeled as a Markov Decision Process (MDP).

The study of preference-based planning is motivated by existing probabilistic planning with temporal logic constraints. In these planning problems, the desired behavior of the system is first specified by a temporal logic formula [2], and then the agent synthesizes a policy that either maximizing the probability of satisfying the formula [3], [4], or incorporating the formula as constraints for probabilistic planning [5], [6], or treating the satisfaction of the formula as one objective in multi-criteria planning [7]. However, a major limitation is that if the specification is not satisfiable or satisfied with a small probability, then a new specification will be generated and the process iterates. The lack of flexibility in specifying "what needs to be satisfied" motivates the development of the minimum violation planning concept: Starting with a user-defined preference relation to prioritized properties, minimum violation planning in a deterministic system [8] decides which low-priority constraints to be violated. A related topic to minimal violation planning is called shield synthesis [9], [10], where a shield performs run-time policy revision to ensure that the system satisfies a

\footnotetext{
$1 \mathrm{Jie}$ Fu is with the Department of Robotics Engineering, Worcester Polytechnic Institute, 01520, Worcester, USA. j fu2 @ wpi . edu

This material is based upon work supported by the Air Force Office of Scientific Research under award number FA9550-21-1-0085.
}

small set of critical properties, such as state-dependent safety conditions. Automated specification-revision is proposed in [11] where the formula can be revised with a cost and the planning problem is formulated into a multi-objective MDP that trades off minimizing the cost of revision and maximizing the probability of satisfying the revised formula. Robust and recovery specifications are introduced by[12] and pre-specify what behaviors are expected when the part of the system specification (i.e. , the environment assumption) fails to be satisfied. Minimal-violation planning, revision, and shield synthesis are all informed with preferences. In current work, a preference is either represented by a utility function [13], determined by prioritizing sub-specifications [8], [14], a distance measure for the deviation from the original formula [11], or a set of hard constraints for run-time verification. Yet, utilitarian representations and prioritization cannot generalize to general ordinal preferences, which can describe pre-orders over possible outcomes. In AI community, preference-based planning is studied and aims to solve the most preferred plan as a sequence of actions (see a survey [15]). In [16], the authors proposed a logical language for specifying preferences over the evolution of states and actions associated with a deterministic plan.

Motivated by existing work, we develop a model of preferences over temporal goals. This model allows the agent to express its preference as a pre-order of sets of temporal evolving trajectories, rather than a total order. Based on the proposed model, we solve a class of time-constrained preference-based planning, in which the agent must maximally satisfy its preference within a pre-defined finite time duration. The planning method is based on encoding the satisfaction of preference formulas into the objective function and constraints of a Mixed-Integer Linear Programming (MILP). By solving the MILP, we obtain a finite-horizon policy that maximizes the preference satisfaction (defined formally in Sec. III). We use a running example to explain the proposed preference specification languages and discuss the limitation in its expressiveness. Finally, we employ a stochastic gridworld example to show the outcomes of the preference-based planning methods given different preference specifications.

\section{PRELIMINARY}

In this section, we define the notations used throughout the paper and the model of the stochastic system.

Notation: Given a finite set $X$, let $\mathcal{D}(X)$ be the set of probability distributions over $X$. Let $\Sigma$ be an alphabet (a finite set of symbols). Given $k \in \mathbb{Z}_{+}, \Sigma^{k}$ indicates a set of words with length $k, \Sigma^{\leq k}$ indicates a set of words with 
length no greater than $k$, and $\Sigma^{0}=\lambda$ is the empty word. $\Sigma^{*}$ is the set of finite words (also known as the Kleene closure of $\Sigma$ ), and $\Sigma^{\omega}$ is the set of infinite words. Given a set $X$, let $\mathbf{1}_{X}$ be the indicator function with $\mathbf{1}_{X}(x)=1$, if $x \in X$ and 0 otherwise.

We consider the stochastic system is modeled by a labeled MDP [17]. A labeled MDP is a tuple $M=$ $\langle S, A, \nu, P, \mathcal{A P}, L\rangle$, where $S$ and $A$ are finite state and action sets, $\nu \in \mathcal{D}(S)$ is the initial state distribution, the transition probability function $P(\cdot \mid s, a) \in \mathcal{D}(S)$ is defined as a probability distribution over the next state given action $a$ is taken at state $s, \mathcal{A P}$ denotes a finite set of atomic propositions, and $L: S \rightarrow 2^{\mathcal{A P}}$ is a labeling function mapping each state to the set of atomic propositions true in that state. We defer the definition of policies to Section IV

\section{Specifying Preferences over Temporal GoAls}

In this section, we introduce an automata-theoretic model of preferences over temporal goals.

Definition 1 (Preferences of temporal goals). The model of preferences of temporal goals is represented by a finite-state, deterministic automaton with preferences over acceptance conditions, formally,

$$
\mathcal{A}=\left\langle Q, 2^{\mathcal{A P}}, \delta, q_{0}, \mathfrak{M}\right\rangle,
$$

where

- $Q$ is a set of states.

- $2^{\mathcal{A P}}=\Sigma$ is the alphabet.

- $\delta: Q \times \Sigma \rightarrow Q$ is a deterministic transition function, which maps a state $q$ and an input symbol $\sigma \in \Sigma$ to a unique next state.

- $q_{0}$ is the initial state.

- $\mathfrak{M}=\langle\mathrm{APF}, \varphi\rangle$ is a preference model, where

- APF is a set of atomic preference formulas. A preference formula is atomic if it can be expressed as the following form $X_{0} \preccurlyeq X_{1} \preccurlyeq \ldots \preccurlyeq X_{n}$ for some $X_{i} \subseteq Q$ and $X_{i} \cap X_{j}=\emptyset$, for any $i \neq j, 0 \leq i, j \leq 1$. The formula is said to have a length $n$, which is the number of operators $\preccurlyeq$ in the formula. The relation $\preccurlyeq$ is reflexive and transitive (a so-called "preorder") over $2^{Q}$ and its strict sub-relation $\prec$ is given by:

$$
X \prec X^{\prime} \text { iff } X \preccurlyeq X^{\prime} \wedge X^{\prime} \nprec X .
$$

$\preccurlyeq$ is said to be total iff for all $X, X^{\prime}$, either $X \preccurlyeq X^{\prime}$ or $X^{\prime} \preccurlyeq X$.

- $\varphi$ is a generalized preference formula (GPF) and is constructed from APF using propositional logic. A GPF $\varphi$ is an atomic preference formula or one of the following two forms

(1) $\psi_{1} \& \psi_{2} \ldots \& \psi_{n}$ [General And].

(2) $\psi_{1}\left|\psi_{2} \ldots\right| \psi_{n}$ [General Or].

where $n \geq 1$ and each $\psi_{i}$ is a GPF.

The "General And" (\&) and "General Or" (|) follow from [16] and are different from logical $\wedge$ and $\vee$ that can be used to define atomic preference. Because $X_{1} \preccurlyeq X_{2} \wedge X_{2} \preccurlyeq X_{3}$ is equivalently an APF $X_{1} \preccurlyeq X_{2} \preccurlyeq X_{3}$ for the transitivity of the preference relation. But $\varphi_{1} \& \varphi_{2}$ means the combination of two different preferences. This will be clarified later as we introduce the value of preference satisfaction (see Def. 2).

Remark 1. Note that the negation of an atomic preference is not defined, because the negation of " $X$ is preferred to $X^{\prime \prime}$ " does not mean that " $X$ ' is preferred to $X$ ". This is because if $X$ is not preferred to $X^{\prime}$, then it can be one of the three possible cases: 1) $X^{\prime}$ is preferred to $X$;2) $X$ and $X^{\prime}$ are indifferent, or 3) $X$ and $X^{\prime}$ are incomparable.

The preference model $\mathcal{A}$ can be used to specify preferences over subsets of words in $\Sigma^{*}$ where $\Sigma=2^{\mathcal{A P}}$. To see this, we first consider a regular Deterministic Finite Automaton (DFA), that is, $A=\left\langle Q, \Sigma, \delta, q_{0}, X\right\rangle$ with a reachability acceptance condition: a word $w \in \Sigma^{*}$ is accepted by $A$ if $\delta\left(q_{0}, w\right) \in X$. Now, given two sets $X \subseteq Q$ and $X^{\prime} \subseteq Q$ and the states and transitions of the automaton $\mathcal{A}$, we can derive two sets of words: $\mathcal{L}(X)$ is the set of words accepted by $\left\langle Q, \Sigma, \delta, q_{0}, X\right\rangle$ and $\mathcal{L}\left(X^{\prime}\right)$ is the set of words accepted by $\left\langle Q, \Sigma, \delta, q_{0}, X^{\prime}\right\rangle$. The atomic preference $X \preccurlyeq X^{\prime}$ means a word in $\mathcal{L}\left(X^{\prime}\right)$ is preferred to any words in $\mathcal{L}(X)$. The interpretation of APF $X_{0} \preccurlyeq X_{1} \preccurlyeq \ldots \preccurlyeq X_{n}$ means a word in $\mathcal{L}\left(X_{i}\right)$ is preferred to any words in $\mathcal{L}\left(X_{j}\right)$ for $0 \leq j<i$, for $1 \leq i \leq n$.

We now use a running example to illustrate the proposed preference model.

Example 1. Consider a robot motion planning example, the robot needs to sequentially visit a set of regions, denoted $A, B$ and $C$. The preference of the human supervisor is stated informally as follows:

- P-A: The more regions being visited the better.

- P-B: If only two regions are visited, he prefers visiting region $\{A, B\}$ to visiting either regions $\{B, C\}$, or $\{A, C\}$.

- P-C: If all three regions can be visited, he prefers to visit $A, B$ first and then $C$. And visiting three regions (regardless of ordering) is better than visiting two regions.

We first construct the automaton in Fig. 1. The automaton is understood as follows: State 0 means no regions has been reached. State 1,2,3 means that the agent has visited $A, B, C$, respectively. State $4,5,6$ means that the agent has visited two of the regions. State 7 means that the agent has visited all three regions. The atomic preferences are

- $\left(P_{0, X}\right) X \succ \emptyset$ for all $X \subseteq Q \backslash\left\{q_{0}\right\}$;

- $\left(P_{1}\right)\{7\} \succcurlyeq\{4,6\}$;

- $\left(P_{2}\right)\{7\} \succcurlyeq\{5\}$;

- $\left(P_{3}\right)\{5\} \succcurlyeq\{4,6\}$;

- $\left(P_{4}\right)\{4,6\} \succcurlyeq\{1,2,3\}$;

- $\left(P_{5}\right)\{7\} \succcurlyeq\{4,6,5\} \succcurlyeq\{1,2,3\} \succcurlyeq\{0\}$;

It is clear that P-A is expressed by the APF $P_{5}$ and P-B is expressed by APF $P_{3}$. However, P-C cannot be defined using the automaton in Fig. 1 because the set of states in automaton cannot distinguish the set of words that visiting $\{A, B\}$ and then $C$. To express the last preference, we 
refine the automaton by re-partitioning the Myhill-Nerode equivalence relation [18] over $\Sigma^{*}$ defined by the structure of the automaton in Fig. 1 to generate a new automaton in Fig. 2 With the new automaton, we add a new atomic preference $P_{6}:=\{8\} \succcurlyeq\{7\}$ and duplicate the original $P_{2}$ as $P_{2}^{\prime}:=\{8\} \succcurlyeq\{5\}$. The last preference is then expressed as

- P-C: $P_{1} \& P_{2}^{\prime} \& P_{6}$.

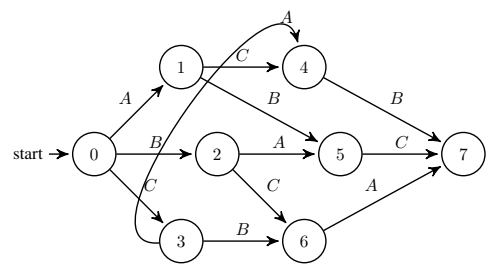

Fig. 1: The automaton for defining preferences.

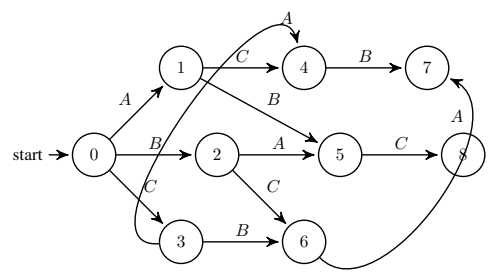

Fig. 2: The refined automaton for defining preferences.

Remark 2. In our formulation, we directly express the preferences over acceptance conditions in an automaton. This can be related to preferences over temporal logic formulas as follows: Given the states and transitions of a DFA, with different final state sets, the automaton accepts different languages, i.e. subsets of $\Sigma^{*}$. Each subset $\mathcal{L}$ of $\Sigma^{*}$ can be associated with a temporal logic formula such that a word is in the set $\mathcal{L}$ if and only if the word satisfies the said formula. Conversely, if we have two formulas $\varphi_{1}$ and $\varphi_{2}$ such that the word satisfying $\varphi_{1}$ is preferred to any word satisfying $\varphi_{2}$, we can construct the union product of $\mathcal{A}_{1}$ (expressing $\varphi_{1}$ ) and $\mathcal{A}_{2}$ (expression $\varphi_{2}$ ), and then define the preferences over subsets of states in the union product. The GPF would enable us to specify preference, such as, $\varphi_{1}$ is preferred to $\varphi_{2}$ and $\varphi_{3}$ is preferred to $\varphi_{2}$ but $\varphi_{1}$ and $\varphi_{3}$ are incomparable. Constructing automated algorithms to translate preferences over temporal logic formulas to our preference models of temporal goals is our ongoing work.

In a stochastic system, each preference formula can be satisfied to some degree. We define the value of preference satisfaction as follows.

Definition 2 (Value of Preference Satisfaction). Given a stochastic process $\left\{Y_{t}, t \geq 0\right\}$ with a stopping time defined on the probability space $(\Sigma, \mathcal{F}, P)$, the value of preference satisfaction $v: \Phi \rightarrow[0,1]$ is defined as follows

- For each atomic preference $p \in \operatorname{APF}$ and $p:=X_{0} \preccurlyeq$

$$
\begin{aligned}
& X_{1} \preccurlyeq X_{2} \ldots \preccurlyeq X_{n}, \\
& v(p)=\left\{\begin{array}{cc}
\operatorname{Pr}\left(X_{i}\right) & \text { if } \exists i>0, \operatorname{Pr}\left(X_{i}\right) \geq \operatorname{Pr}\left(X_{i-1}\right) \\
0 & \forall k \geq i, \operatorname{Pr}\left(X_{k}\right)<\operatorname{Pr}\left(X_{k-1}\right) \\
0 & \text { otherwise. }
\end{array}\right.
\end{aligned}
$$

where $\operatorname{Pr}(Z) \triangleq \operatorname{Pr}\left(\left\{w \in \Sigma^{*} \mid \delta\left(q_{0}, w\right) \in Z\right\}\right)$ is the probability of terminating in set $Z$ upon reading the word sampled from the stochastic process.

- For GPF $\varphi_{1} \& \varphi_{2}, v(\varphi)=\min \left(v\left(\varphi_{1}\right), v\left(\varphi_{2}\right)\right)$.

- For GPF $\varphi_{1} \mid \varphi_{2}, v(\varphi)=\max \left(v\left(\varphi_{1}\right), v\left(\varphi_{2}\right)\right)$.

Here the stopping time is a random variable that determines when to stop the process. With a stopping time, the sampled word is of a finite length.

Next, we provide some important remarks about the expressiveness of the proposed preference models:

- Conditional preferences: A conditional preference means if some condition is satisfied, then the preference is expressed. For example, if the fuel is still sufficient after visiting two regions $A, B$, then the preference of visiting region $D$ over $C$ is expressed. If the fuel is insufficient after visiting $A, B$, then the agent is indifferent to $C$ and $D$ and wishes to visit one more region before running out of fuel. To express such conditional preferences, we would need to introduce quantitative formulas and cost/reward functions, which is out of the scope for this paper. The current definition of preferences can capture qualitative conditional preferences. For example, if $A, B$ is visited, then $D$ is preferred to $C$. This preference can be expressed by first defining the set $L_{1}$ of words ending in $D$ after seeing only $A, B$, and a set $L_{2}$ of words ending in $C$ after seeing only $A, B$, and construct the DFA that accepts $L_{1} \cup L_{2}$. We can define the preferences over state sets in the constructed DFA to express the conditional preference.

- The quality of satisfaction: Consider the stochastic process $\left\{Y_{t}, t \geq 0\right\}$ and two preference formulas $\varphi_{1}$ and $\varphi_{2}$. In some cases, it is desirable to say that if the value $v\left(\varphi_{1}\right)$ is below a given threshold, then the agent's preference is $\varphi_{2}$, otherwise, the agent's preference is $\varphi_{1}$. This preference cannot be expressed with the given model as it requires the generalization of the model with quantitative formulas.

\section{Preference-based Probabilistic Planning WITH TIME CONSTRAINTS}

Preference satisfaction is often coupled with limited resources (monetary or time). In this section, we consider a class of planning problems with preferences and time constraints. First, to reason about system dynamics and preferences over temporal sequences, we use the product operation [17] to augment the state space of a labeled MDP with automaton states.

Definition 3 (Product MDP). Given a labeled MDP $M=\langle S, A, \nu, P, \mathcal{A P}, L\rangle$, a DFA with preference $\mathcal{A}=$ 
$\left\langle Q, \Sigma, \delta, q_{0}, \mathfrak{M}\right\rangle$, the product of $M$ and $\mathcal{A}$ is denoted by

$$
\mathcal{M}=M \otimes \mathcal{A}=\langle S \times Q, A, \bar{\nu}, \Delta\rangle,
$$

with (1) a set $S \times Q$ of states, (2) the set $A$ of actions, (3) a distribution over the initial states $\bar{\nu}\left(s_{0}, \delta\left(q_{0}, L\left(s_{0}\right)\right)=\nu\left(s_{0}\right)\right.$, (3) the transition function defined by $\Delta\left(\left(s^{\prime}, q^{\prime}\right) \mid(s, q), a\right)=$ $P\left(s^{\prime} \mid s, a\right) \mathbf{1}_{\left\{q^{\prime}\right\}}\left(\delta\left(q, L\left(s^{\prime}\right)\right)\right)$.

In the product MDP, a finite-horizon, nonstationary, stochastic policy in the MDP is a sequence $\pi=\mu_{0} \mu_{1} \ldots \mu_{T}$ where $\mu_{t}: S \times Q \rightarrow \mathcal{D}(A)$ that maps a state $(s, q)$ into a distribution over actions at time $t$, for some $0 \leq t \leq T$. Given a product MDP $\mathcal{M}$ and a policy $\pi$, the policy induces a stochastic process $\mathcal{M}^{\pi}=\left\{\left(S_{t}, Q_{t}\right) \mid t=0, \cdots, T\right\}$ where $S_{t}$ and $Q_{t}$ are the random variables for the $t$-th MDP state and automaton states, respectively. It holds that $\left(S_{t+1}, Q_{t+1}\right) \sim \Delta\left(\cdot \mid\left(S_{t}, Q_{t}\right), A_{t}\right)$ and $A_{t} \sim \mu_{t}\left(S_{t}, Q_{t}\right)$. We say the stochastic process $\mathcal{M}^{\pi}$ is policy $\pi$-induced.

Definition 4 (Finite-Horizon Preference Satisfaction). Given a Markov chain $\mathcal{M}^{\pi}$ and a GPF $\varphi$, the value of preference satisfaction given a finite time bound $T$, denoted $v(\varphi, \pi, T)$, is defined to be the value of $\varphi$ given the stochastic process $\left\{L\left(S_{t}\right), 0 \leq t<T\right\}$ where $S_{t}$ is the $t$-th MDP state in $\mathcal{M}^{\pi}$.

The problem of preference-based planning with time constraint is formally stated as follows.

Problem 1. Given a labeled MDP $M$, a preference model $\mathcal{A}$, a GPF $\varphi$, and a finite time $T$, compute a policy $\pi=$ $\mu_{0} \mu_{1} \ldots \mu_{T-1}$ that maximizes the value $v_{\varphi}$ (see Def. (2) for satisfying the preference formula within $T$ time steps. Formally, $\pi^{*}=\arg \max _{\pi} v(\varphi, \pi, T)$.

To solve Problem 11 we combine time-constrained MDP planning with the semantics of preferences to formulate a mixed integer linear program (MILP). However, it is noted that an atomic preference with length greater than 1 will introduce a lexicographic constraint and requires iterative search. In the scope of this work, we consider a subclass of preference models where the atomic preferences are of length 1 and the solution vith MILP.

Decision variables We introduce a set of continuous variables $\{y(t,(s, q), a) \mid t=0,1, \ldots, T-1,(s, q) \in S \times Q, a \in$ $A\}$ where $y(t,(s, q), a)$ is interpreted as the probability of visiting state $(s, q)$ in the process $\mathcal{M}^{\pi}$ and taking action $a$ at time $t$, for $t=0, \ldots, T$. The variables satisfy the following two constraints:

$$
\begin{aligned}
& \sum_{a \in A} y(0,(s, q), a)=\bar{\nu}(s, q), \quad \forall(s, q) \in S \times Q, \\
& \sum_{a \in A} y\left(t,\left(s^{\prime}, q^{\prime}\right), a\right)= \\
& \sum_{a \in A} \sum_{(s, q) \in S \times Q} \Delta\left(\left(s^{\prime}, q^{\prime}\right) \mid(s, q), a\right) y(t-1,(s, q), a) \\
& \forall t: 1 \leq t \leq T-1, \forall\left(s^{\prime}, q^{\prime}\right) \in S \times Q .
\end{aligned}
$$

where (2) enforces the constraint from the initial state distribution and (3) means that the probability of reaching $\left(s^{\prime}, q^{\prime}\right)$ at time $t$ is the same as the sum of probabilities of reaching some other state at time $t-1$ from which an action is chosen to reach $\left(s^{\prime}, q^{\prime}\right)$ next. These two constraints are generated based on the linear program formulation for constrained MDPs [19].

Encoding of Preferences Given a GPF $\varphi$, we introduce a Boolean variable $z_{\varphi}$, which equals to 1 if there exists a policy $\pi$ that $v(\varphi, \pi, T)>0$. We recursively generate the MILP constraints corresponding to $\varphi$. The time $T$ is omitted with the understanding that the following constraint generation is applicable for time-constrained planning.

1) Atomic Preferences of Length 1 Each atomic preference $p:=\left(X \preccurlyeq X^{\prime}\right)$ of length 1 is associated with a value $v_{p} \in$ $[0,1]$, subject to the following constraint:

$$
\begin{aligned}
& v_{p}-y\left(T, X^{\prime}\right) \geq M \cdot\left(z_{p}-1\right), \\
& v_{p}-y\left(T, X^{\prime}\right) \leq 0, v_{p} \geq 0, v_{p} \leq M \cdot z_{p}, \\
& y\left(T, X^{\prime}\right)-y(T, X) \leq M \cdot z_{p}-\epsilon\left(1-z_{p}\right) \\
& y\left(T, X^{\prime}\right)-y(T, X) \geq m\left(1-z_{p}\right)+\epsilon z_{p}
\end{aligned}
$$

where $y(T, X)=\sum_{q \in X} \sum_{a \in A} y(T,(s, q), a)$ is the probability of reaching the set $X$ at time $T, M>0$ is the upper bound on $y\left(T, X^{\prime}\right)-y(T, X)$, which is one, and $m$ is the lower bound on $y\left(T, X^{\prime}\right)-y(T, X)$, which is -1 , and $\epsilon>0$ is a constant close to zero. The last two constraint means if $y\left(T, X^{\prime}\right) \geq y(T, X)$ then $z_{p}=1$, else $z_{p}=0$. The first and second constraints mean that $v_{p}=y\left(T, X^{\prime}\right)$ when $z_{p}=1$. The third and fourth constraints mean that $v_{p}=0$ if $z_{p}=0$. The strong preference $X \prec X^{\prime}$ is expressed using the same set of constraints. This is because when $z_{p}=1$, the last constraint enforces $y\left(T, X^{\prime}\right)>y(T, X)$ when $\epsilon>0$.

2) Boolean operation We now encode \& (general and) and | (general or) of atomic preferences as mixed-integer linear constraints. Let an atomic preference $p$ have its corresponding variable $v_{p}$. The "generalized and" of atomic preferences $\varphi:=p_{1} \& p_{2}$ generates the following constraints:

$$
\begin{aligned}
& v_{\varphi} \leq v_{p_{i}}, \quad i=1,2 \\
& v_{\varphi}-v_{p_{1}} \geq m \cdot(1-z), \\
& v_{\varphi}-v_{p_{2}} \geq m \cdot z \\
& v_{p_{1}}-v_{p_{2}} \leq M \cdot z-\epsilon(1-z)
\end{aligned}
$$

where $z$ is a Boolean variable such that when if $v_{p_{1}} \geq v_{p_{2}}$ then $z=1$. The first three lines of constraints enforce $v_{\varphi}=$ $\min \left(v_{p_{1}}, v_{p_{2}}\right)$. The "generalized or" of atomic preferences $\varphi:=p_{1} \mid p_{2}$ generates the following constraints,

$$
\begin{aligned}
& v_{\varphi} \geq v_{p_{i}}, \quad i=1,2 \\
& v_{\varphi} \leq v_{p_{1}}+M \cdot(1-z), \\
& v_{\varphi} \leq v_{p_{2}}+M \cdot z, \\
& v_{p_{1}}-v_{p_{2}} \leq M \cdot z-\epsilon(1-z),
\end{aligned}
$$

where $z$ is a Boolean variable such that when if $v_{p_{1}} \geq v_{p_{2}}$ then $z=1$. The first three lines of constraints enforce $v_{\varphi}=\max \left(v_{p_{1}}, v_{p_{2}}\right)$. For general conjunction/disjunction of more than one atomic preference formulas, we can apply the constraint generation recursively. 
Finally, for a given GPF $\varphi$, we obtain a set of constraints $\left\{f_{i}(\mathbf{v}, \mathbf{y}, \mathbf{z}) \geq 0, i=1, \ldots k\right\}$ where $\mathbf{z}$ is a vector of Boolean variables, $\mathbf{y}$ is the vector of decision variables, and $\mathbf{v}$ is the vector of values of sub-formulas in the GPF. The MILP formulation for time-constrained preference-based planning is shown as follows:

$$
\begin{aligned}
& \max _{\mathbf{y}, \mathbf{v}, \mathbf{z}} v_{\varphi} \\
& \text { s.t.: }(2),(3), f_{i}(\mathbf{v}, \mathbf{y}, \mathbf{z}) \geq 0, i=1, \ldots k \\
& \quad \mathbf{z} \in\{0,1\}^{\ell}, \mathbf{v} \succeq 0, \mathbf{y} \succeq 0 .
\end{aligned}
$$

where $k, \ell$ are appropriate numbers generated from the set of constraints, and $\succeq$ is the inequality symbol for element-wise no less than. The solution to the MILP defines the optimal policy of Problem 11. For each $t$ such that $0 \leq t \leq T-1$, the decision rule is

$$
\begin{aligned}
\mu_{t}((s, q), a):=\frac{y(t,(s, q), a)}{\sum_{a^{\prime}} y\left(t,(s, q), a^{\prime}\right)}, & \\
& \forall(s, q) \in S \times Q \text { and } \forall a \in A .
\end{aligned}
$$

It is noted that for time-constrained planning, the optimal policy is time-dependent [20].

Remark 3. In [16], the authors introduces aggregated preferences. One such preference is lexicographical preference, written as $\operatorname{lex}\left(\varphi_{1}, \ldots, \varphi_{n}\right)$ where each $\varphi_{i}$ is a preference. This preference means there is a lexicographic ordering over preference formulas. Only if $\varphi_{i}$ is not satisfiable, then the agent should try to satisfy $\varphi_{i+1}$, for $i=1, \ldots, n-1$. To generate policies given a lexicographical preferences, we can use multiple rounds of preference-based planning given GPFs $\varphi_{1}, \ldots, \varphi_{n}$, following the lexicogrphical ordering. The computation is terminated when the first satisfiable GPF and its corresponding optimal policy are found.

Complexity: The planning method given the automataencoding of preferences has the same complexity as MILPs, which are NP-hard. As the optimal policy is time-dependent, the planning state space is the Cartesian product of $S, Q$, and $\{t \mid T-1 \geq t \geq 0\}$. Thus, the computation can be expensive when the formula is complex and has a long time horizon.

\section{CAse Study}

Continuing with the preferences specified in Example 1 let's consider the planning problem in a stochastic grid world as shown in Fig. 3 . For agent's different action (heading north ('N'), south ('S'), west ('W') and east ('E')), the probability of arriving the intended cell is shown in Table I. In Fig. 3, blue disk represents the agent whose initial cell is 10, the green cells are regions of interests, with the labeling: $L(0)=A, L(12)=B$, and $L(22)=C$, and the red cells are obstacles. If the agent reaches an obstacle, then it will stay in the cell for all future time. We consider the same automaton in Fig. 1] with preferences over a set of temporal goals.

\begin{tabular}{c|cccc} 
Action & $\mathrm{N}$ & $\mathrm{E}$ & $\mathrm{S}$ & $\mathrm{W}$ \\
\hline & & & & \\
'N' & 0.8 & 0.1 & 0 & 0.1 \\
'E' & 0.05 & 0.8 & 0.15 & 0 \\
'S' & 0 & 0.1 & 0.7 & 0.2 \\
'W' & 0.15 & 0 & 0.15 & 0.7
\end{tabular}

TABLE I: The probabilistic dy-

namics. Fig. 3: A $5 \times 5$ gridworld
We first consider three preference specifications: 1$) \varphi_{1} \triangleq$

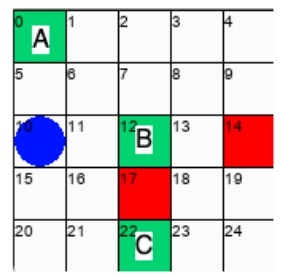
$P_{1}$, 2) $\varphi_{2} \triangleq P_{1} \& P_{4}$, and 3) $\varphi_{3} \triangleq P_{1} \mid P_{4}$ (see the definition of preferences in Example 1). We vary the bound $T$ on the total time steps. Figure 4 shows the optimal value of $v(\varphi, \pi, T)$ under the optimal policy for $\varphi \in\left\{\varphi_{1}, \varphi_{2}, \varphi_{3}\right\}$.

In Fig. 4a the green triangles (resp. blue squares) represent the probabilities of ending in $S \times\{4,6\}$ (resp. $S \times\{7\})$ given different time limits $T$ for $5 \leq T \leq 24$ under the computed policies. The red dashed line represents the values of satisfaction for $\varphi=P_{1}$ at different $T$. When $T$ is small $\leq 10$, the agent cannot visit all three regions to reach the automaton state 7 , thus, the value of satisfaction is zero. When $T \geq 11$, the agent can satisfy the preference. The value of satisfaction increases monotonically as $T$ increases. There are some small probabilities $(\leq 0.002)$ of ending in $S \times\{4,6\}$ when $T \geq 11$.

In Fig. 4b and Fig. 4c the green triangles (resp. blue squares) represent the values of $P_{1}$ (resp. $P_{4}$ ) under the optimal policies. The red dashed line represents the values of satisfaction for $\varphi=P_{1} \& P_{4}$ (resp. $\varphi=P_{1} \mid P_{4}$ ) given different $T$. In the case of conjunction (Fig. $4 \mathrm{~b}$ ), it is observed that the value of satisfaction is lower than that for the case of disjunction (Fig. 4c). This is understandable, as to satisfy both $P_{1}$ and $P_{4}$, the agent is required to ensure, when the time stops, it will has a larger chance of visiting two regions than one, and at the same time, ensures the probability of visiting three regions is greater than that of visiting two.

Comparing Fig. 4c and Fig. 4a it is noted that in the case of disjunction, the values of $P_{1}$ are all zeros (blue squares) at different time bounds $T$. This is because for any $T>0$, the value of satisfying $P_{4}$ is greater than the value of satisfying $P_{1}$ given the optimal policy. Due to the disjunction, the agent satisfies $\varphi_{3}$ by satisfying $P_{4}$ for $T \geq 6$.

Next, we consider a more complex specification $\varphi:=\mathrm{P}-\mathrm{C}$ which says that the agent prefers to reach region $A, B$ first and then $C$ if all three regions can be visited. We use the automaton in Fig. 2 which distinguishes a set of words that contains $A, B$ before seeing a $C$ from the rest of words containing all three symbols. The result is shown in Fig. 4d, the red, dashed line is the values of satisfying P-C under different time bounds. The sequences of green triangles are the values of $P_{1}$ given the optimal policy, which coincide the values of $\mathrm{P}-\mathrm{C}$ given the same policy under different $T$. The blue dotted line is the sum $v\left(P_{1}\right)+v\left(P_{2}^{\prime}\right)$ under the optimal policy. The sum is the probability of visiting all three regions in the policy-induced stochastic process. Before time step 12 , the specification is not satisfiable and the values are zero. This is because the added constraint, $\{8\} \succcurlyeq\{7\}$, is not satisfiable when $T \leq 12$. 

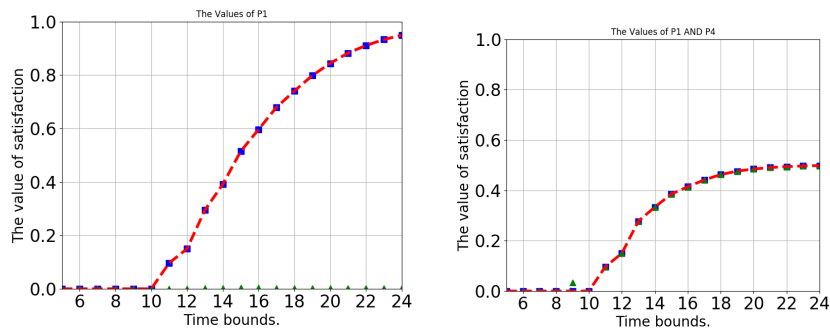

(a) The value of satisfaction for $\varphi_{1}$.
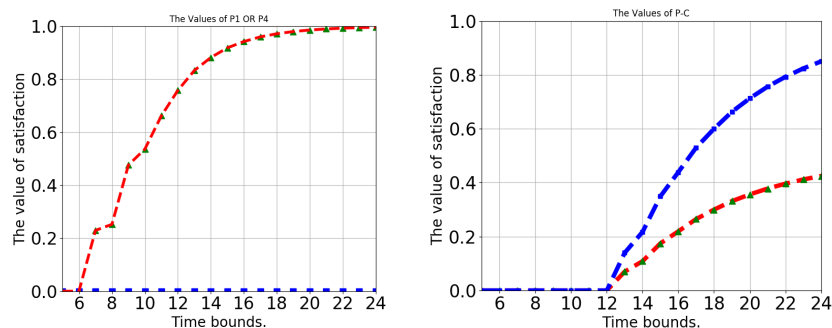

(c) The value of satisfaction for $\varphi_{3}=P_{1} \mid P_{4}$. (d) The value of satisfaction for P-

C : $P_{1} \& P_{2}^{\prime} \& P_{6}$.

Fig. 4: The values of satisfaction given different preference formulas and different time bounds.

From the experiment, we can observe a major difference in preference-based planning and probabilistic planning with temporal logic constraints [17], [4], [3]. If the goal is to simply reaching all three regions, then the optimal policy should be generated by optimally satisfying a preference formula $\{7\} \succcurlyeq\{0\}$ in DFA (Fig. 11) based on Def. 2, which would attain a value close to 1 as $T$ increases to infinity. With preferences, other than a constraint that requires the probability of visiting three regions is greater than that of visiting none, we can incorporate more flexible constraints, for example, visiting $k$ regions is preferred to visiting $j$ when the time runs out for any $k>j$.

Experiments are conducted using Python MIP toolbox [21] on a MacBook with $1.6 \mathrm{GHz}$ Intel Core i5 and $8 \mathrm{~GB}$ Memory. The maximal CPU time for running all experiments is $28 \mathrm{sec}$ (for $T=24$ and preference formula P-C).

\section{CONClusion And Future Work}

In this paper, we presented a way to specify preferences over temporal goals and a synthesis method to generate the optimal policy for the value of preference satisfaction in a stochastic system. This preference model supports preferences over temporally extended goals and is built on the relation between automata theory and preferences in deterministic planners [16]. Building on this work, we will further investigate the planning algorithms for more general atomic preferences (with length greater than one). We will study the specifications of temporally evolving preferences, generalized conditional preferences, and their corresponding algorithms in probabilistic and stochastic systems.

\section{REFERENCES}

[1] R. Hastie and R. M. Dawes, Rational choice in an uncertain world: The psychology of judgment and decision making. Sage, 2010.

[2] Z. Manna and A. Pnueli, The temporal logic of reactive and concurrent systems: Specification. Springer Science \& Business Media, 2012.

[3] X. C. Ding, S. L. Smith, C. Belta, and D. Rus, "Mdp optimal control under temporal logic constraints," in 2011 50th IEEE Conference on Decision and Control and European Control Conference. IEEE, 2011, pp. 532-538.

[4] M. Hasanbeig, Y. Kantaros, A. Abate, D. Kroening, G. J. Pappas, and I. Lee, "Reinforcement learning for temporal logic control synthesis with probabilistic satisfaction guarantees," in 2019 IEEE 58th Conference on Decision and Control (CDC). IEEE, 2019, pp. 5338-5343.
[5] B. Lacerda, D. Parker, and N. Hawes, "Optimal and dynamic planning for markov decision processes with co-safe ltl specifications," in 2014 IEEE/RSJ International Conference on Intelligent Robots and Systems, 2014, pp. 1511-1516.

[6] M. Wen, R. Ehlers, and U. Topcu, "Correct-by-synthesis reinforcement learning with temporal logic constraints," in 2015 IEEE/RSJ International Conference on Intelligent Robots and Systems (IROS). IEEE, 2015, pp. 4983-4990.

[7] J. Fu and U. Topcu, "Synthesis of Shared Autonomy Policies With Temporal Logic Specifications," vol. 13, no. 1, pp. 7-17, Jan. 2016.

[8] J. Tumova, G. C. Hall, S. Karaman, E. Frazzoli, and D. Rus, "Leastviolating control strategy synthesis with safety rules," in Proceedings of the 16th international conference on Hybrid systems: computation and control. ACM, 2013, pp. 1-10.

[9] R. Bloem, B. Könighofer, R. Könighofer, and C. Wang, "Shield synthesis," in International Conference on Tools and Algorithms for the Construction and Analysis of Systems. Springer, 2015, pp. 533548.

[10] M. Alshiekh, R. Bloem, R. Ehlers, B. Könighofer, S. Niekum, and $\mathrm{U}$. Topcu, "Safe reinforcement learning via shielding," The ThirtySecond AAAI Conference on Artificial Intelligence, 2018.

[11] M. Lahijanian and M. Kwiatkowska, "Specification revision for Markov decision processes with optimal trade-off," in Proc. 55th Conference on Decision and Control (CDC'16), 2016, pp. 7411-7418.

[12] R. Bloem, H. Chockler, M. Ebrahimi, and O. Strichman, "Synthesizing reactive systems using robustness and recovery specifications," in 2019 Formal Methods in Computer Aided Design (FMCAD). IEEE, 2019, pp. 147-151.

[13] K. V. Hindriks and M. B. Van Riemsdijk, "Using temporal logic to integrate goals and qualitative preferences into agent programming," in International Workshop on Declarative Agent Languages and Technologies. Springer, 2008, pp. 215-232.

[14] T. Tomita, A. Ueno, M. Shimakawa, S. Hagihara, and N. Yonezaki, "Safraless LTL synthesis considering maximal realizability," Acta Informatica, vol. 54, pp. 655-692, 2017.

[15] J. A. Baier and S. A. Mcllraith, "Planning with Preferences," AI Magazine, vol. 29, no. 4, p. 25, 2008. [Online]. Available: https://www.aaai.org/ojs/index.php/aimagazine/article/view/2204

[16] M. Bienvenu, C. Fritz, and S. A. Mcllraith, "Specifying and computing preferred plans," Artificial Intelligence, vol. 175, no. 7-8, pp. 13081345, May 2011.

[17] C. Baier and J.-P. Katoen, Principles of model checking. MIT press, 2008.

[18] J. E. Hopcroft, R. Motwani, and J. D. Ullman, Introduction to Automata Theory, Languages, and Computation (3rd Edition). USA: Addison-Wesley Longman Publishing Co., Inc., 2006.

[19] E. Altman, Constrained Markov decision processes. CRC Press, 1999, vol. 7.

[20] M. Mundhenk, J. Goldsmith, C. Lusena, E. Allender, N. M. Mundhenk, and N. J. Goldsmith, Complexity of Finite-Horizon Markov Decision Process Problems, 2000.

[21] S. T.A.M, H. G. and Toffolo, "Mip: Python tools for Modeling and Solving Mixed-Integer Linear Programs (MIPs)," 2020. 